\title{
Cloning improvements suggested
}

The recent birth of three generations of cloned mice from an adult somatic cell by scientists at the University of Hawaii (Honolulu) suggests that cloning, or nuclear transfer and reprogramming of mammalian cells, is both possible and reproducible in species other than the sheep. Although the Hawaiian group has demonstrated the most successful technique to date, many insist the technology still has far to go before it is commercially viable. Despite several improvements to the nuclear transfer procedure, numerous questions remain unanswered.

"Cloning mice had been thought to be nearly impossible due to the fragility of their oocytes," says senior scientist Ryuzo Yanagimachi, the Hawaiian-team leader. But their rate of success using nuclear transfer to produce offspring is $1-2 \%$, about double that of Ian Wilmut's team when his team created Dolly the sheep. The improvement may be due to modifications of Wilmut's method. These include microinjection instead of electrofusion of the donor nucleus to the recipient egg, choice of donor cells that are normally arrested in G0 phase of the cell cycle instead of using a cell population that has to be induced to arrest in $\mathrm{G} 0$ in vitro, and waiting three to six hours before activating the newly created couplets.

"While we know how to do nuclear transfer with adult somatic cells, we don't know how to do it efficiently," said Ian Wilmut at the recent Second Annual Congress on Mammalian Cloning in Washington, DC in late June. "There are losses at every stage of development of the embryos, as well as serious congenital malformations in many animals."

This was echoed by a number of speakers at the meeting, who noted that "a great number" of animals born from nuclear transfer have been born missing organs such as kidneys and hearts, and that many have also been stillborn or born greatly oversized. "Through our current methods of nuclear transfer, we don't really know what kind of embryos we are creating-normal or not," said Tanja Dominko, staff scientist in Gerald Schatten's laboratory at the Oregon Regional Primate Research Center (Beaverton), and who used to work on bovine cloning at the University of Wisconsin.

According to Dominko, relatively low birthrates and other problems may be due to current methods used in nuclear transfer, which she thinks are too harsh and damaging to newly created embryos. "We need to go back and address these questions before we go much farther-especially in nonhuman primates, for ethical and financial reasons," she says.

Dominko recommends further modifications to steps in the nuclear transfer process: Rather than using ultraviolet light and dyes to illuminate the DNA in the recipient egg's cytoplasm, Dominko suggests using longer wavelengths of light, such as green or red, which in turn could be kept on longer than the 20-30 seconds needed to confirm enucleation. "If UV light damages our DNA, it is likely it is also damaging the oocyte's, especially mitochondrial DNA that is left in the oocyte after enucleation," she says. Removing less cytoplasm during enucleation could also make a difference.

Instead of using an electrical pulse to fuse donor nucleus and recipient cytoplasm, she suggests following Yanagimachi's method and employing microinjection of the nucleus into donor egg. This does two things: It avoids high-field strength electrical pulsing for fusion, and eliminates cytoplasmic contribution of the donor cell.

For activation of the egg, current techniques use harsh approaches such as electric shock, cyclohexamide (a protein synthesis inhibitor), or treatment with DMAP (dimethyl amino purine; a protein kinase inhibitor), the last two shut down all protein synthesis and protein kinases, respectively, rather than selectively targeting specific proteins. Dominko suggests using ionomycin, a calcium transient inducer, "which mimics what sperm do," she says. Alternatively, one could use sperm factor-an uncharacterized extract from sperm that induces the same changes in an egg but without adding DNA.

Yanagimachi's group activated their metaphase-arrested mouse eggs with strontium after injecting the nucleus, in an arrested state, into the cytoplasm; Wilmut's group put the nucleus, after serum starving it, into an activated egg, tricking it into "thinking" it had been fertilized. But Dominko thinks that timing of activation is the crucial element.

"Perhaps a specific cell cycle stage for donor nucleus-G0-is necessary but it alone is clearly not sufficient," says Robert Wall, research physiologist at the US Department of Agriculture (Beltsville, MD).

Steven Stice, chief scientific officer of Advanced Cell Technology (Worcester, MA), is working on transgenic cloning of cows. Although he has observed pregnancy losses at 45 days due to a "placental effect" (edema in 11 of 13 bovine pregnancies), he does not think these abnormalities are only due to nuclear transfer. "While there is room for improvement in the technique, nuclear transfer is still much more efficient than [the older microinjection method] for commercial purposes."

With such modifications in mind, Dominko and the rest of Schatten's team plan to begin the first nuclear transfer experiments in primates this autumn.

Vicki Brower
Enrich cell lines for high producers or rare cells in one day, using gelmicrodrop single cell encapsulation and FACS.

When time to

market matters, drop everything and inquire about our lease program or screening service today. READER INQUIRY NO. 1104 617-868-2399 www.onecell.com 